\title{
ATC Safety Assessment Based on Fuzzy Matter-element with Weighting by AHP and Entropy Method
}

\author{
Ping'an Wang ${ }^{1, a}$, Shiyao Ren $^{2, b}$, Nan Yang ${ }^{2, c}$ \\ ${ }^{1}$ Xijing College, Xi’an, Shaanxi, 710123, China \\ ${ }^{2}$ Air Traffic Control and Navigation College, Air Force Engineering, University, Xi’an, Shaanxi, 710051, \\ China \\ aawangpa02@163.com, brensy888@sina.com, c yangn001@126.com
}

Keywords: Air Traffic Control; Fuzzy Matter-element; Weighting; Safety Assessment

\begin{abstract}
To prevent the air collision accident caused by Air Traffic Control (ATC), according to the, an ATC safety assessment method based on fuzzy matter-element analysis with combination weighting is proposed. In the method, firstly, the safety assessment index system of ATC system is established, then the combination weighting by Analytic Hierarchy Process and Entropy method is conducted for assessment index, finally the fuzzy matter-element method is used to evaluate the operational safety of ATC system. The example analysis shows that the proposed method is feasible and effective.
\end{abstract}

\section{Safety assessment index system for ATC system}

To establish a safety assessment index system that reflects the actual operation of Air Traffic Control (ATC) system, it is necessary to follow the basic principles of science, comprehensiveness and hierarchy. On the basis of extensive research on relevant cases, 43 influencing factors are selected through consulting experts and combining actual operation of ATC system. The 10 influencing factors with cumulative contribution rate are initially selected to construct the safety assessment index system of the ATC system, that is, C1: Failure rate of technical examination; C2: Operation violation rate; C3: Coordination of ATC group; C4: Error frequency of ATC command; C5: Failure rate of maintenance inspection; C6: Technical standard oversight rate; C7: Weather condition during operation; C8: Traffic volume in ATC area; C9: Enforcement of rules and regulations; C10: Smooth information communication.

\section{Fuzzy matter-element model}

The fuzzy matter-element analysis method [1] was proposed in 1995 by Professor Xiao Fangjun which combines the matter-element analysis theory and the fuzzy set theory to effectively deal with the fuzzy incompatibility problem in the assessment process. And because of its excellent performance, it is widely used in safety assessment of ecology, geology, water and construction. In this paper, a fuzzy matter-element analysis model with combination weighting is applied to the safety assessment of ATC system. The assessment process is shown in Figure 1.

\section{Weighting Based on AHP}

AHP is a hierarchical system analysis method, which is more formally focused on the combination of qualitative and quantitative analysis [2]. The basic idea is to decompose a more complex problem into several constituent components, and group the decomposed constituent components according to the dominance relationship, construct an ordered hierarchical structure, and determine the relative importance of each factor in hierarchical structures by means of pairwise comparison. Finally, the judgment of the experts is used to determine the total order of the relative importance of each factor in 
decision-making. The realization steps of AHP are as follows: establish a hierarchical structure model; construct a pairwise comparison matrix; determine the weight vector and perform consistency check.

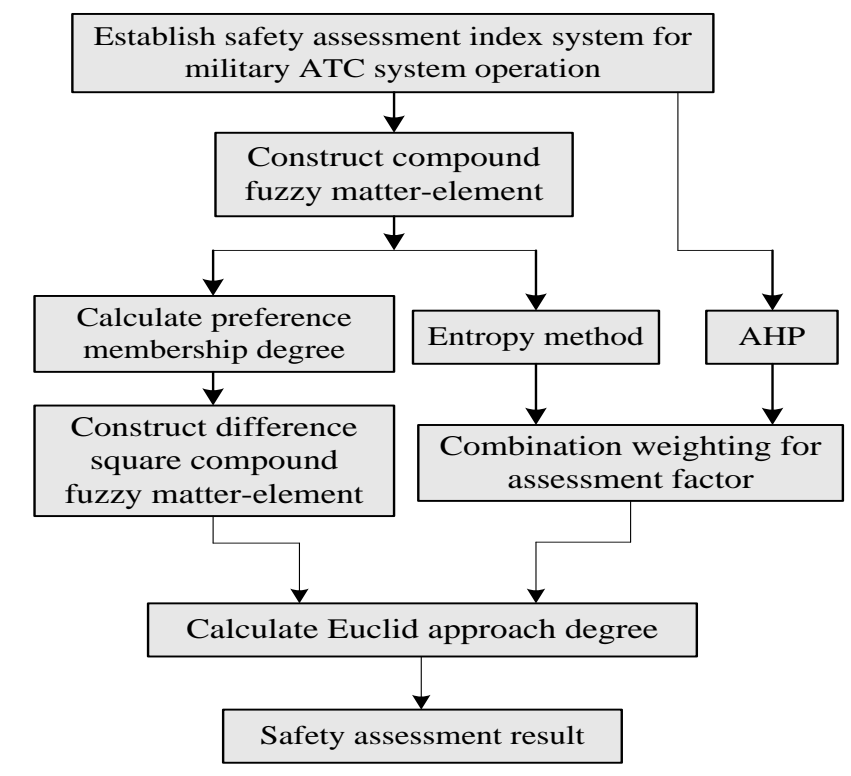

Figure 1 Flow chart of fuzzy matter-element model

\section{Weighting Based on Entropy method}

Entropy is mainly used to describe uncertainty in information theory. The smaller the amount of information, the greater the uncertainty, the larger the entropy; the larger the amount of information, the smaller the uncertainty and the smaller the entropy. According to this characteristic, the entropy method can be used to judge the order degree of the obtained information and its utility[3]. Therefore, the entropy method is regarded as an objective weighting method in the paper. The specific calculation process is as follows:

(1) For $n$ assessment indexs and $m$ things, the following judgment matrix can be established.

$$
R=\left(x_{i j}\right)_{m n}, i=1,2, \cdots, m ; j=1,2, \cdots, n
$$

(2) Normalization process can conducted to obtain a matrix $B$ by the following formula

$$
b_{i j}=\frac{x_{i j}-x_{\min }}{x_{\max }-x_{\min }}
$$

where $x_{\max }$ is the most satisfactory in different things under a certain index; $x_{\min }$ is the most unsatisfactory in different things under a certain index.

(3) According to the definition of entropy in information theory, the entropy of $n$ indexs of $m$ things can be expressed as

$$
H_{i}=\frac{\sum_{j=1}^{m} f_{i j} \ln f_{i j}}{\ln m}
$$

where $i=1,2, \ldots, m ; j=1,2, \ldots, n . f_{i j}=b_{i j} / \sum_{j=1}^{m} b_{i j}$. Obviously, when $f_{i j}$ is equal to zeros, $\ln f_{i j}$ is meaningless, so the formula can be improved as 


$$
f_{i j}=\frac{1+b_{i j}}{\sum_{j=1}^{m}\left(1+b_{i j}\right)}
$$

(4) Calculate the entropy weight of the assessment index

$$
\beta_{i}=\frac{1+H_{i}}{n-\sum_{i=1}^{n} H_{i}} \text {, and satisfy } \sum_{i=1}^{n} \beta_{i}=1
$$

\section{Combination Weighting}

According to the fuzzy matter-element analysis process, to correctly evaluate the operation safety of ATC system, weighting is a very critical issue. AHP determines the weight according to the analyst's preference for each assessment factor, which is simple and easy to implement, but is subject to subjective factors. The entropy method determines the weight according to the correlation and variation degree of the assessment factor itself, effectively avoiding the influence of human factors, but is vulnerable to interference with random errors in the sample. In the safety assessment of ATC operation, the paper combines the two and complements each other. On the basis of the principle of minimum discriminant information, we propose to combine the weights obtained by AHP and entropy method to obtain the combined weights, which can strive to achieve the subjective and objective unity for weight determination[4].

MDI is a very important concept in entropy theory, describing the difference between two probability distributions, which was proposed by Good et al in the middle of the last century. Suppose that $\alpha_{i}$ is the subjective weight of $i$-th index obtained by AHP, $\beta_{i}$ is the objective weight of $i$-th index obtained by entropy method, and $w_{i}$ denotes the combination weight of $i$-th index. According to the theory of authentication information, the combination weight $w_{i}$ should be as close as possible to $\alpha_{i}$ and $\beta_{i}$, therefore, the objective function can be established as follows:

$$
\begin{aligned}
\min & F=\sum_{i=1}^{n} w_{i}\left[\ln \frac{w_{i}}{\alpha_{i}}\right]+\sum_{i=1}^{n} w_{i}\left[\ln \frac{w_{i}}{\beta_{i}}\right] \\
\text { s.t. } & \sum_{i=1}^{n} w_{i}=1 \text {, and } w_{i}>0
\end{aligned}
$$

Calculate the minimum by Lagrange multiplier method

$$
w_{i}=\frac{\sqrt{\alpha_{i} \beta_{i}}}{\sum_{i=1}^{n} \sqrt{\alpha_{i} \beta_{i}}}
$$

\section{Example analysis}

After establishing the safety assessment index system of ATC system operation and expounding the fuzzy matter assessment process, it can be combined with the actual operation of the ATC system to verify the feasibility and effectiveness of the model.

The overall safety status of 5 ATC areas is evaluated combinationly, and 10 experts in ATC safety were selected to score the assessment indexs C3, C5, C7, C8 and C10 that could not directly obtain data. The percentage system can be adopted, that is, the scoring range is $[0,100]$, which is invalid beyond this range. Finally, the scores of each index in 5 ATC areas given by the experts and collected are averaged, obtaining raw data is shown in Table 1 , and thus constitutes a composite fuzzy matter-element. Calculate Euclid approach degree of 5 ATC areas according to formula (13). The calculation results are shown in Table 3. At the same time, according to Euclid approach degree, the 
sorting of safety assessment of 5 ATC areas can be obtained.

According to the research on the actual operation of ATC system, the safety assessment index C1, C2, C4, C6, and C9 are as small as possible; The index C3, C5, C7, C8, and C10 are as large as possible. The calculated values of the two are combined to obtain the preference membership fuzzy matter-element.

The assessment data corresponding to 10 assessment indices of 5 ATC areas is used to calculate the objective weights of 10 assessment indices by entropy method. The comparison judgment matrix can be constructed according to the expert information, and consistency check of AHP is conducted, calculating the subjective weights. Finally, the combination weights are calculated based on MDI. The combination weights are shown in Table 2.

$$
R_{5,10}=\left[\begin{array}{ccccc}
0.12 & 0.10 & 0.14 & 0.07 & 0.09 \\
0.04 & 0.02 & 0.05 & 0.01 & 0.02 \\
80 & 78 & 85 & 81 & 92 \\
3 & 7 & 6 & 4 & 5 \\
92 & 90 & 98 & 94 & 95 \\
0.01 & 0.05 & 0.02 & 0.04 & 0.01 \\
85 & 95 & 80 & 90 & 85 \\
75 & 80 & 85 & 80 & 92 \\
0.05 & 0.02 & 0.04 & 0.07 & 0.03 \\
94 & 91 & 92 & 90 & 92
\end{array}\right]^{\mathrm{T}} \quad \quad_{5,10}=\left[\begin{array}{ccccc}
0.29 & 0.57 & 0.00 & 1.00 & 0.71 \\
0.25 & 0.75 & 0.00 & 1.00 & 0.75 \\
0.14 & 0.00 & 0.50 & 0.21 & 1.00 \\
1.00 & 0.00 & 0.25 & 0.75 & 0.50 \\
0.25 & 0.00 & 1.00 & 0.50 & 0.63 \\
1.00 & 0.00 & 0.75 & 0.25 & 1.00 \\
0.33 & 1.00 & 0.00 & 0.67 & 0.33 \\
0.00 & 0.29 & 0.59 & 0.29 & 1.00 \\
0.40 & 1.00 & 0.60 & 0.00 & 0.80 \\
1.00 & 0.25 & 0.50 & 0.00 & 0.50
\end{array}\right]^{\mathrm{T}}
$$

The safety assessment order of 5 ATC areas is: ATC Area 5> ATC Area 1> ATC Area 4> ATC Area 3> ATC Area 2, of which ATC Area 5 is the safest.

Table 1 Raw data of safety assessment for each ATC area

\begin{tabular}{cccccc}
\hline Index & Area 1 & Area 2 & Area 3 & Area 4 & Area 5 \\
\hline$C_{1}$ & 0.12 & 0.10 & 0.14 & 0.07 & 0.09 \\
$C_{2}$ & 0.04 & 0.02 & 0.05 & 0.01 & 0.02 \\
$C_{3}$ & 80 & 78 & 85 & 81 & 92 \\
$C_{4}$ & 3 & 7 & 6 & 4 & 5 \\
$C_{5}$ & 92 & 90 & 98 & 94 & 95 \\
$C_{6}$ & 0.01 & 0.05 & 0.02 & 0.04 & 0.01 \\
$C_{7}$ & 85 & 95 & 80 & 90 & 85 \\
$C_{8}$ & 75 & 80 & 85 & 80 & 92 \\
$C_{9}$ & 0.05 & 0.02 & 0.04 & 0.07 & 0.03 \\
$C_{10}$ & 94 & 91 & 92 & 90 & 92 \\
\hline
\end{tabular}

Table 2 Combination weights of safety assessment indices for each ATC area

\begin{tabular}{|c|c|c|c|}
\hline Index & AHP & $\begin{array}{r}\text { Entropy } \\
\text { method } \square\end{array}$ & $\begin{array}{c}\text { Comprehensive } \\
\text { weight } w\end{array}$ \\
\hline$C_{1}$ & 0.1221 & 0.0923 & 0.1102 \\
\hline$C_{2}$ & 0.0876 & 0.1075 & 0.0956 \\
\hline$C_{3}$ & 0.1195 & 0.1074 & 0.1147 \\
\hline$C_{4}$ & 0.0793 & 0.0958 & 0.0859 \\
\hline$C_{5}$ & 0.0954 & 0.0902 & 0.0933 \\
\hline$C_{6}$ & 0.1280 & 0.1402 & 0.1329 \\
\hline$C_{7}$ & 0.0971 & 0.0908 & 0.0946 \\
\hline$C_{8}$ & 0.0865 & 0.0922 & 0.0887 \\
\hline$C_{9}$ & 0.1204 & 0.0956 & 0.1104 \\
\hline$C_{10}$ & 0.0641 & 0.0879 & 0.0737 \\
\hline
\end{tabular}

\section{Conclusions}

The fuzzy matter-element method is used to combinationly evaluate the operational safety of ATC system. In the method, it is important to weight the indices. For this, on the basis of subjective weight calculated by AHP and objective weight calculated by entropy method, a combination weighting method is proposed based on minimum discriminant information. It show that, proposed method is more scientific and reasonable for ATC safety assessment, and the result is more realistic. 


\section{References}

[1] B. Zhang, Q. D. Yong, F. C. Xiao. Fuzzy matter-element analysis. Beijing: Petroleum Industry Press, 1997.

[2] J. E. Chang, T. L. Jiang. Research on the weight of coefficient through analytic hierarchy process. Journal of WUT (Information and Management Engineering), 29(1), (2007), 153-156.

[3] P. Mu, P. Ai. Application of entropy weight and attribute recognition model in assessment of water conservancy modernization. Journal of Economics of Water Resources, 29(5), (2011), 1-4.

[4] X. J. Wang, P. Ai, Q. Y. Ding. Application of fuzzy matter-element model based on combined weights in assessment of water resources modernization. Journal of China Three Gorges University (Natural Science), 37(2), (2015), 1-5. 